\title{
Health Information and Monitoring of Sexually Transmitted Infections (SIM Study): a Randomized Controlled Trial Protocol for Enhancing Adherence to Syphilis Treatment and Follow-up
}

Eliana Marcia Ros Wendland ( $\square$ eliana.wendland@hmv.org.br)

Hospital Moinhos de Vento

Vanessa M. de Oliveira

Moinhos de Vento Hospital: Hospital Moinhos de Vento https://orcid.org/0000-0002-9770-9993

Flavia Souza

Ministério da Saúde: Ministerio da Saude

Gerson Pereira

Ministério da Saúde: Ministerio da Saude

Antonio Gerbase

Hospital Moinhos de Vento

\section{Research Article}

Keywords: Syphilis, clinical trial, telemonitoring, treatment adherence, protocol

Posted Date: December 21st, 2021

DOI: https://doi.org/10.21203/rs.3.rs-694253/v1

License: (c) (1) This work is licensed under a Creative Commons Attribution 4.0 International License.

Read Full License 


\section{Abstract}

Background:Syphilis has recently resurfaced as a significant public health problem. Since the 2000s, isolated syphilis outbreaks have increasingly occurred in North America, Europe, and Australia; in Brazil, there have been progressive increases in both congenital and acquired syphilis. There are several possible explanations, such as misdiagnosis of acquired syphilis, which could increase the number of untreated transmitters in the population; failure to initiate or complete treatment; and nontreatment of sexual partners (leading to reinfection). Mobile technologies have been successfully used to promote behaviour changes and can positively impact treatment and follow-up adherence in patients with infectious diseases. The purpose of this clinical trial is to evaluate treatment and monitoring methods in patients with syphilis, including follow-up by telephone, via a game in a smartphone app and at public health centres.

Methods: The SIM study is a single-centre, randomized controlled trial with a 12-month follow-up period. The aim is to identify the most effective method of follow-up regarding patient compliance with treatment. The tests will be performed in a mobile unit in easily accessible locations. The goal is to perform 10,000 rapid tests for syphilis. Patients with a confirmed diagnosis according to VDRL tests will be randomized to one of three arms: telephone, smartphone game, or conventional in-person follow-up. All analyses will follow the intention-to-treat principle.

Conclusion: If we find differences in effectiveness, a major change in the conventional approach for this patient population may be needed, potentially affecting current Brazilian health policy strategies.

\section{Short Summary}

Syphilis has re-emerged as a significant public health problem.

Our study tests new options adapted to current technological advances to increase adherence to treatment and disease monitoring.

\section{Introduction}

Syphilis infection has recently re-emerged as a significant public health problem, with an increasing incidence in many countries. ${ }^{1}$ Syphilis is a bacterial infection caused by Treponema pallidum; transmission occurs through sexual contact (acquired syphilis) or during pregnancy (vertical transmission) and can easily be prevented through condom use. ${ }^{2}$ The primary lesion (primary syphilis) frequently goes unnoticed, and many individuals are asymptomatic during the early stages of infection. Congenital syphilis, on the other hand, occurs when a mother infected with syphilis transmits the infection to her child during pregnancy; in these cases, the disease has a poor prognosis, and the risks of spontaneous abortion, foetal death and premature infant death are increased. ${ }^{3}$ 
Beginning in the 2000s, a growing number of isolated syphilis outbreaks were reported in North America, Europe, and Australia, primarily within sexual networks of men who have sex with men (MSM); syphilis infection is often associated with HIV co-infection and co-transmission. ${ }^{4}$ While the number of cases in specific groups has increased in high-income countries, in low-middle-income countries, syphilis remains an endemic disease, affecting the general population. ${ }^{5}$

In Brazil, there has been a progressive increase in both congenital and acquired syphilis in recent years. Data from compulsory reports indicated that acquired syphilis rates increased from 2.0 cases/100,000 inhabitants in 2010 to 58.1 cases/100,000 inhabitants in 2017. Congenital syphilis also increased substantially (3.6-fold) from 2.4 to 8.6 cases per 1,000 live births. Although these increases occurred in all Brazilian regions, some geographical regions had rates above the Brazilian average, including the Southern Region, in which the city of Porto Alegre had an incidence rate of congenital syphilis 3.8 times higher than the rate in the Brazilian general population. ${ }^{6,7}$

Several causes could be implicated in the increasing number of cases, such as misdiagnosis of acquired syphilis, which could lead to a larger number of untreated transmitters in the population; failure to initiate or complete treatment; nontreatment of sexual partners (leading to reinfection); or lack of adequate prenatal care, as syphilis is most often diagnosed during pregnancy screening or during screening for other sexually transmitted infections. ${ }^{7}$

Mobile technologies and strategies to track test results and appointments have been successfully used as tools to promote behaviour changes and can positively impact adherence to treatment and follow-up in patients with chronic ${ }^{8-11}$ and infectious diseases. ${ }^{12-15}$

There are 8.3 billion mobile phone subscriptions worldwide; this number is greater than the global population, and the numbers of subscribers in low- and middle-income countries are increasing rapidly, increasing opportunities to easily connect with a large number of patients to provide individual-level support. ${ }^{16}$ Herein, we describe the study protocol for a parallel randomized controlled trial to evaluate different home telemonitoring technologies to enhance adherence to treatment and follow-up in syphilis patients.

\section{Materials And Methods}

\section{Study design}

The SIM study is a randomized controlled trial including three parallel arms: (1) follow-up with telephone calls, (2) follow-up using gamification in a mobile app, and (3) follow-up with usual care provided by the public health system. Randomization will occur at the individual level.

\section{Participants and recruitment}


All adults aged 18 years and older will be invited to answer a questionnaire to collect socio-economic and sexual behaviour data and undergo a rapid test for syphilis, HIV and hepatitis B and C in a mobile unit located in an area accessible to a large population. The unit will have different attractive features, such as free internet, charging areas for smartphones, and shaded and rest areas with screens, to increase the participation rate. Participants with a positive rapid test will be invited to undergo confirmatory testing. This protocol was written following the Standard Protocol Items: Recommendations for Interventional Trials (SPIRIT) statement to define the clinical trial protocol.

\section{Eligibility criteria}

The study population will consist of people living in the Porto Alegre area who undergo a confirmatory test and return to obtain their results (run-in). Participants who do not return after three contact attempts will be excluded from the study, as well as pregnant women, participants who are not able to provide contact information, participants who are illiterate and those who underwent syphilis treatment within the previous three months.

\section{Sample size}

Considering a confidence level of $95 \%$, an incidence rate of 93.7 per 100,000 inhabitants in the municipality of Porto Alegre, ${ }^{11}$ and an acceptable difference of 60.0 per 100,000 inhabitants, it is estimated that testing 10,000 individuals will be necessary to obtain an accurate prevalence of syphilis in the municipality. This sample will serve as the basis for the inclusion of patients in the clinical trial.

For the randomized clinical trial, another sample size calculation will be considered. Using a previous article in which $16.5 \%$ of the participants who were diagnosed with syphilis received appropriate treatment and $55.9 \%$ received inappropriate treatment as a reference, it is expected that at least half of the latter group will eventually receive proper treatment, for a total of $44.45 \%$ receiving appropriate treatment. ${ }^{17}$ Thus, considering a $95 \%$ confidence level, $80 \%$ power and a maximum loss rate of $20 \%, 51$ participants in each study group will be needed.

\section{Study Procedures}

A paper-based questionnaire to collect sociodemographic, behavioural and health information will be administered to all participants to check eligibility, and a rapid test for syphilis will be performed. All participants with a positive test result will undergo confirmatory testing. Upon the receipt of confirmatory test results, the participants will be invited to take part in the clinical trial, and a web-based questionnaire to collect information such as age, sex, social class, education level, alcohol consumption, tobacco and drug use, risk for syphilis, history of hepatitis and other sexually transmitted infections (STIs), number of sexual partners, type of sex and condom use.

\section{Randomization and blinding}


Patients will be randomized at a ratio of 1:1:1 among the three intervention arms: follow-up by telephone, follow-up using a smartphone game, or follow-up with usual care in the public health system. Random allocation will be performed in blocks with variable sizes, stratified by participants with and without smartphone access. Randomization will be performed using R software. Individuals who have access to a smartphone will be randomly assigned to any of the 3 groups, while individuals without access to a smartphone will be randomly assigned to one of only two groups: the telephone follow-up group or the control group.

An independent statistician will be responsible for computerised randomization ( $R$ software) but will not be involved in the determination of participant eligibility, assessment or implementation of the interventions. Blinding will be applied to minimise bias. The health professionals who conduct the baseline and initial assessments will be blinded to intervention allocations. Researchers responsible for the follow-up by telephone intervention will not have access to performance and evaluation data. Only the biostatistician will have access to the data stored in the data entry system, minimising investigator influence.

\section{Intervention}

The intervention strategies aim to increase adherence to treatment and monitoring and promote communication with sexual partners (Figure 1). All intervention and conventional strategies followed the guidelines of the Clinical Protocol and Therapeutic Guidelines for Comprehensive Attention to People with Sexually Transmitted Infections of the Ministry of Health of Brazil. ${ }^{4}$

\section{Strategy 1: Follow-up by telephone}

Initial contact will be made 2 days before treatment, on the day of treatment and for 14 days until the next treatment dose. If the patient fails to apply treatment within 14 days of the last treatment dose, the intervention will be considered a failure and the patient will have to restart treatment. For monitoring, contact will be made 7 days before the exam and on the day of the exam to encourage the patient to get tested, and the patient will receive 1 call per week for 3 weeks until the patient takes the VDRL test.

\section{Strategy 2: Follow-up using a game in a smartphone app}

A thematic game will be developed to encourage treatment and monitoring in patients with syphilis. The first phase of the game will be open to all study participants regardless of their rapid test result and will have information about prevention strategies and sexually transmitted infections. In the second phase, the participants will be prompted by notifications via smartphone 2 days before the day of treatment, on the day of treatment and every day for 14 days after the date they should have received the injection. The participant is awarded coins to buy clothing and accessories for his/her avatar if he/she reads the information about STIs and provides information about his/her partners. The participant passes the phase of the game when he/she has taken the medication doses and completed the VDRL test. In 
different game phases, the avatar takes the bus and travels to different capitals of Brazil where syphilis infection rates are also high.

\section{Strategy 3: Conventional follow-up}

The conventional strategy involves compulsory notification by a healthcare professional, and the patient is directed to seek treatment and follow-up at the primary health care unit closest to his or her home. The outcomes will be accessed by reviewing data from the public health records.

\section{Outcome assessment}

Compliance with treatment for those in the mobile app arm will be assessed according to the uploaded game data provided by the participant and use of the app during the study period. Adherence to treatment for those receiving telephone follow-up will be assessed through routine phone calls and questionnaire questions about the date of examination and treatment. Adherence in the usual care arm will be assessed by information collected from the participant's medical record at their referral health unit.

\section{Specimen processing}

Samples will be treated as biohazardous material, and specimen handling will be carried out in a biosafety cabinet. Once arriving in the laboratory, serum samples will be immediately fractionated. Two aliquots will be frozen at $-80^{\circ} \mathrm{C}$ as backup samples. All samples with positivity on the treponemal test (rapid test) will be subjected to a confirmatory nontreponemal test. The aliquot will be directly used for the VDRL and rapid plasma regain (RPR) tests. If the VDRL test is negative, an additional treponemal test will be used to confirm (TPHA) syphilis positivity. An online platform for data entry will be used by the study team to add participant data, rapid test results, biological sample information and exam reports. Data will be monitored daily by the project statistician.

\section{Statistical analyses}

Descriptive analysis will be performed to characterize the study population. The categorical variables will be summarized by absolute frequencies and percentages, while continuous variables will be described as means and standard deviations or medians and interquartile ranges.

To compare proportions, the chi-Square test and Fisher's exact test will be used. Student's t test or the nonparametric Mann-Whitney test will be used to compare continuous variables.

The primary goal of the study is to compare treatment adherence and follow-up among the three parallel study arms. We will investigate the association between treatment adherence and sociodemographic, sexual health and sexual behaviour variables as secondary outcomes. The association will be estimated by calculating crude and adjusted odds ratios (ORs) using logistic regression. Intention-to-treat analysis will be used to assess the clinical effectiveness of the intervention. 
A statistical significance level of 0.05 will be adopted for all statistical analyses. R software, version 4.0.3, will be used for the analysis ${ }^{18}$.

\section{Monitoring and quality control}

During the project development stage, operational manuals were developed along with the questionnaires to serve as general guidelines for conducting interviews, standardizing information and solving general doubts. All researchers involved in the data collection will be trained using simulated interviews and biological sample collections.

A pilot study will be performed for one month to identify and correct problems with the interview, logistical and study procedures.

The participants will be informed about the study results through reports that will be provided at the mobile unit of the study.

Quality control will be performed through system reports, data entry control and data enrolment auditing. During the project development stage, we established written policies for sample management and quality control in the laboratory handbook. All laboratory professionals were trained and certified by the coordinator team according to the study protocol. Those performing the VDRL tests are certified by the National Program of Quality Control (PNCQ).

A web-based platform will be utilized for biological sample tracking and test result recording on a daily basis. Samples will be carefully transported in order to maintain the integrity of the sample, with attention to temperature, preservation methods, special transport containers and time limitations. Once the samples arrive in the laboratory, their label information, quantity and condition for the requested tests will be verified. Frozen specimen aliquots will be tracked using the Freezer Web system. Test results will be photodocumented, and the images will be uploaded to a digital repository.

\section{Ethics and dissemination}

The study was approved by the Research Ethics Board (REB) of the Hospital Moinhos de Vento and Health Secretariat of the Municipality of Porto Alegre. Written consent will be obtained from all participants after they have been informed about study procedures. Data entry will be protective by personal logins and will be available to only the main statistician after the interview is finalized.

Authorship eligibility follows the CRediT guidelines.

\section{Steering Committee}

\section{Dissemination}

The results of the study will be disseminated to the different stakeholders by providing reports to the Ministry of Health and state and federal government agencies, publishing articles in scientific journals 
and presenting the results at scientific conferences and to the Municipal Health Committee, of which users are part of the Single Health System.

\section{Discussion}

Syphilis has been diagnosed in laboratories for over 100 years. Testing is becoming increasingly cheaper, faster, less invasive, and easier. Treatment for this disease has been known for over 60 years, and penicillin is a cheap drug with no major adverse events. However, syphilis has been re-emerging in Brazil and in many countries around the world. The World Health Organization (WHO) estimates that 11 million new cases of syphilis occur each year in Latin America, with 70\% occurring in Brazil.

Open syphilis lesions facilitate HIV transmission. Thus, preventing and treating syphilis could also reduce HIV transmission. Furthermore, co-infection with HIV may change the natural course of syphilis.

The increase in the number of syphilis cases has resulted in new challenges for health management, and new data to guide effective interventions to control the disease are needed. Some questions need to be answered so that better prevention and control strategies can be developed and perhaps generate new health policies. Our study intends to answer the following questions: What is the better e-Health strategy for enhancing treatment and monitoring in patients with syphilis? Will any of the interventions increase the communication with and treatment of sexual partners?

\section{Declarations}

\section{Ethics approval and consent to participate}

The study was approved by the Research Ethics Board (REB) of the Hospital Moinhos de Vento and Health Secretariat of the Municipality of Porto Alegre. Written consent will be obtained from all participants after they have been informed about study procedures. Data entry will be protective by personal logins and will be available to only the main statistician after the interview is finalized.

\section{Consent for publication}

The authors consent for publication of the article.

\section{Availability of data and material}

Quality control will be performed through system reports, data entry control and data enrolment auditing. During the project development stage, we established written policies for sample management and quality control in the laboratory handbook. All laboratory professionals were trained and certified by the coordinator team according to the study protocol. Those performing the VDRL tests are certified by the National Program of Quality Control (PNCQ).

\section{Competing interests}


The authors declare not conflict of interests

\section{Funding}

This work was financed by Hospital Moinhos de Vento through the Program for Supporting the Institutional Development of the Public Health System (PROADI-SUS) and supported by the Brazilian Ministry of Health. The Federal University of Health Sciences of Porto Alegre contributed to the development and application of the laboratory methods.

\section{Authors' contributions}

Study design: Eliana Wendland, Flavia Souza, and Gerson Pereira

Collection: Vanessa Oliveira

Management, analysis, and interpretation of data; Vanessa Oliveira and Eliana Wendland

Analysis and interpretation of data: Luana Pedrotti

Writing of the report and the decision to submit the report for publication: Vanessa Oliveira, Eliana Wendland, Flavia Souza, Gerson Pereira and Antônio Gerbase

\section{Acknowledgements}

Not applicable

\section{Data sharing}

The datasets used and/or analysed during the current study are available from the corresponding author on reasonable request.

\section{Ethics approval}

This research was approved by the Ethics Committee on Human Research of the Moinhos de Vento Hospital research board (Approval No.371.211 / UFCSPA - CAAE: 3.458.634) and by the Health Secretariat of the Municipality of Porto Alegre (SMSPA - CAAE: 3.707.670). All participants will provide written consent after being informed about the content of the study. Our study will be performed in accordance with the Declaration of Helsinki.

\section{Informed consent}

Informed consent forms will be submitted to all patients.

\section{Conflicts of interest}

The authors declare that there are no conflicts of interest. 


\section{Trial registration}

NTC 04753125

Version 1 of protocol 1/09/2020

\section{Trial Status}

Date of recruitment start 2/08/2021

Date recruitment will be completed 12/2022

Clinical trials $0(0)$

Wendland et al.

\section{References}

1. Kitayama K, Segura ER, Lake JE, et al. Syphilis in the Americas: a protocol for a systematic review of syphilis prevalence and incidence in four high-risk groups, 1980-2016. Syst Rev 2017; 6(1): 195.

2. World Health Organization. WHO Guidelines for the Treatment of Treponema Pallidum (Syphilis). Geneva: World Health Organization; 2016.

3. Bowen V, Su J, Torrone E, et al. Increase in incidence of congenital syphilis - United States, 20122014. MMWR Morb Mortal Wkly Rep 2015; 64(44): 1241-1245.

4. Gulland A. Number of cases of syphilis continue to rise. BMJ 2017; 357: J2807.

5. Kojima N, Klausner JD. An update on the global epidemiology of syphilis. Curr Epidemiol Rep 2018; $5(1): 24-38$.

6. Ministério da Saúde, Secretaria de Vigilância em Saúde, Departamento de Vigilância. Manual Técnico Para Diagnóstico da Sífilis. Brasilia, Brazil: Ministério da Saúde; 2016.

7. Ministério da Saúde, Secretaria de Vigilância em Saúde, Departamento de Vigilância. Boletim Epidemiológico de Sífilis. Brasilia, Brazil: Ministério da Saúde; 2017.

8. Adu MD, Malabu UH, Malau-Aduli AEO, et al. Efficacy and acceptability of my care hub mobile app to support self-management in Australians with type 1 or type 2 diabetes. Int $\mathrm{J}$ Environ Res Public Health 2020; 17(7): E2573.

9. Dietrich JJ, Hornschuh S, Khunwane M, et al. A mixed methods investigation of implementation barriers and facilitators to a daily mobile phone sexual risk assessment for young women in Soweto, South Africa. PLoS One 2020; 15(4): e0231086.

10. Kendzor DE, Businelle MS, Waring JJC, et al. Automated mobile delivery of financial incentives for smoking cessation among socioeconomically disadvantaged adults: feasibility study. JMIR Mhealth Uhealth 2020; 8(4): e15960. 
11. Li R, Liang N, Bu F, et al. The effectiveness of self-management of hypertension in adults using mobile health: systematic review and meta-analysis. JMIR Mhealth Uhealth 2020; 8(3): e17776.

12. Yokomitsu K, Irie T, Sekiguchi M, et al. Gamified mobile computerized cognitive behavioral therapy for japanese university students with depressive symptoms: protocol for a randomized controlled trial. JMIR Res Protoc 2020; 9(4): e15164.

13. Huang D, Sangthong R, McNeil E, et al. Effects of a phone call intervention to promote adherence to antiretroviral therapy and quality of life of HIV/AIDS patients in Baoshan, China: a randomized controlled trial. AIDS Res Treat 2013; 2013: 580974.

14. Sabin LL, Bachman DeSilva M, Gill CJ, et al. Improving adherence to antiretroviral therapy with triggered real-time text message reminders: the China adherence through technology study. J Acquir Immune Defic Syndr 2015; 69(5): 551-559.

15. Swendeman D, Ramanathan N, Baetscher L, et al. Smartphone self-monitoring to support selfmanagement among people living with HIV: perceived benefits and theory of change from a mixedmethods randomized pilot study. J Acquir Immune Defic Syndr 2015; 69 Suppl 1(1): S80-S91.

16. You WX, Comins CA, Jarrett BA, et al. Facilitators and barriers to incorporating digital technologies into HIV care among cisgender female sex workers living with HIV in South Africa. Mhealth 2020; 6: 15.

17. Feliz MC, de Medeiros ARP, Rossoni AM, et al. Adherence to the follow-up of the newborn exposed to syphilis and factors associated with loss to follow-up. Rev Bras Epidemiol 2016; 19(4): 727-739.

18. R Core Team (2020). R: A language and environment for statistical computing. R Foundation for Statistical Computing, Vienna, Austria. URL: https://www.R-project.org/.

\section{Figures}




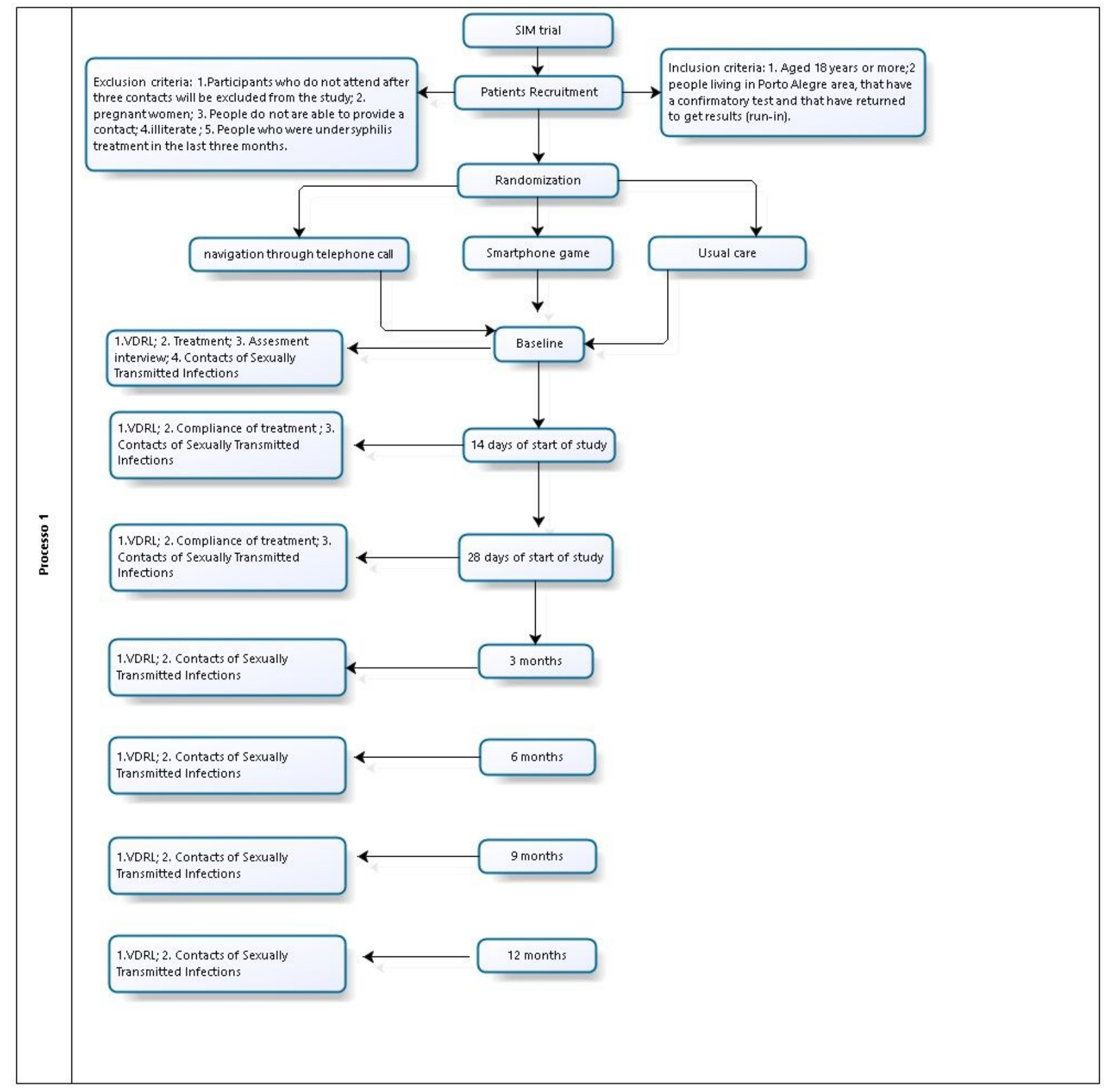

\section{Figure 1}

Profile of study

\section{Supplementary Files}

This is a list of supplementary files associated with this preprint. Click to download. 
- SPIRITFigure1.doc

- 160921SPIRITchecklist.docx

- descriptionofstudyinstruments.docx 